\title{
Supplement zum 5. HeilberufeSCIENCE-Symposium auf dem Interprofessionellen Gesundheitskongress in Dresden
}
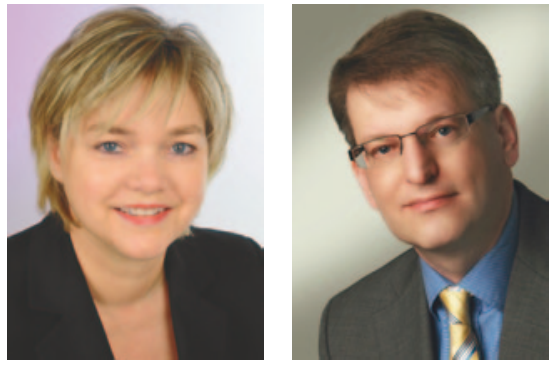

A. Tauchert

J. Klewer

Mit dem 5. HeilberufeSCIENCE-Symposium ist diese wissenschaftliche Veranstaltung wieder nach Dresden „zurückgekehrt“. Wenn die Vorläufer-Symposien seit dem Jahr 2007 mit eingerechnet werden, handelt es sich mittlerweile um die achte Veranstaltung, die dieses Jahr im Rahmen des Interprofessionellen Gesundheitskongresses vom 17.-18.04.2015 stattfindet. Aufgrund der gestiegenen Zahl an eingereichten Abstracts konnte der Zeitumfang des HeilberufeSCIENCE-Symposiums ausgeweitet werden. Somit werden dieses Jahr mehr wissenschaftliche Vorträge $\mathrm{zu}$ einem breiten Themenspektrum aus den Gebieten Pflegemanagement, Pflegepädagogik, Pflegewissenschaften, Gerontologie und Gesundheitswissenschaften/Public Health sowie der Altenhilfe und Altenpflege präsentiert und diskutiert.
Dieses HeilberufeSCIENCE-Supplement beinhaltet alle Abstracts der Vorträge und Poster, die im Rahmen des 5. HeilberufeSCIENCE-Symposiums präsentiert werden, in einer zitierfähigen Form. Alle Abstracts können Sie nach dem Kongress jederzeit auch von den HeilberufeSCIENCESeiten im Portal SpringerLink abrufen. Dort finden Sie auch die regulären Ausgaben von HeilberufeSCIENCE, Informationen zur Einreichung von Beiträgen, zum blinded peerreview-Verfahren und zu den Listungen, u. a. in CINAHL ${ }^{\circledR}$ Complete.

An dieser Stelle möchten wir Ihnen die Lektüre dieses Supplements empfehlen, um im Umfeld des 5. HeilberufeSCIENCE-Symposiums einzelne Inhalte zu vertiefen! Natürlich würden wir uns freuen, wenn wir Sie am 17. und 18. April auf dem Interprofessionellen Gesundheitskongress in Dresden begrüßen dürfen.

Ihre

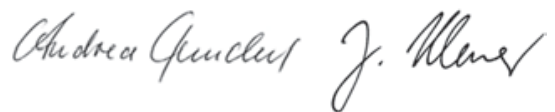

A. Tauchert - J. Klewer 